\title{
A minimally processed dietary pattern is associated with lower odds of metabolic syndrome among Lebanese adults
}

\author{
Lara Nasreddine ${ }^{1,2}$, Hani Tamim ${ }^{3,4}$, Leila Itani ${ }^{5}$, Mona P Nasrallah ${ }^{2,6}$, \\ Hussain Isma'eel ${ }^{2,7}$, Nancy F Nakhoul ${ }^{2,6}$, Joana Abou-Rizk' and Farah Naja ${ }^{1, *}$ \\ 'Department of Nutrition and Food Sciences, Faculty of Agricultural and Food Sciences, American University of \\ Beirut, PO Box 1 1-0236, Riad El Solh 1107 2020, Beirut, Lebanon: ${ }^{2}$ Vascular Medicine Program, American \\ University of Beirut, Beirut, Lebanon: ${ }^{3}$ Clinical Research Institute, Faculty of Medicine, American University of Beirut \\ Medical Center, Beirut, Lebanon: ${ }^{4}$ Department of Internal Medicine, Faculty of Medicine, American University of \\ Beirut Medical Center, Beirut, Lebanon: ${ }^{5}$ Department of Nutrition \& Dietetics, Faculty of Health Sciences, Beirut Arab \\ University, Beirut, Lebanon: ${ }^{6}$ Department of Internal Medicine, Division of Endocrinology, Faculty of Medicine, \\ American University of Beirut Medical Center, Beirut, Lebanon: ${ }^{7}$ Department of Internal Medicine, Division of \\ Cardiology, Faculty of Medicine, American University of Beirut, Beirut, Lebanon
}

Submitted 11 0ctober 2016: Final revision received 10 July 2017: Accepted 12 July 2017: First published online 2 0ctober 2017

\begin{abstract}
Objective: To (i) estimate the consumption of minimally processed, processed and ultra-processed foods in a sample of Lebanese adults; (ii) explore patterns of intakes of these food groups; and (iii) investigate the association of the derived patterns with cardiometabolic risk.

Design: Cross-sectional survey. Data collection included dietary assessment using an FFQ and biochemical, anthropometric and blood pressure measurements. Food items were categorized into twenty-five groups based on the NOVA food classification. The contribution of each food group to total energy intake (TEI) was estimated. Patterns of intakes of these food groups were examined using exploratory factor analysis. Multivariate logistic regression analysis was used to evaluate the associations of derived patterns with cardiometabolic risk factors.

Setting: Greater Beirut area, Lebanon.

Subjects: Adults $\geq 18$ years ( $n$ 302) with no prior history of chronic diseases.

Results: Of TEI, 36.53 and $27 \cdot 10 \%$ were contributed by ultra-processed and minimally processed foods, respectively. Two dietary patterns were identified: the 'ultra-processed' and the 'minimally processed/processed'. The 'ultra-processed' consisted mainly of fast foods, snacks, meat, nuts, sweets and liquor, while the 'minimally processed/processed' consisted mostly of fruits, vegetables, legumes, breads, cheeses, sugar and fats. Participants in the highest quartile of the 'minimally processed/processed' pattern had significantly lower odds for metabolic syndrome $(\mathrm{OR}=0 \cdot 18,95 \%$ CI 0.04, 0.77), hyperglycaemia $(\mathrm{OR}=0 \cdot 25$, $95 \%$ CI $0.07,0.98)$ and low HDL cholesterol (OR=0.17, $95 \%$ CI 0.05, 0.60).

Conclusions: The study findings may be used for the development of evidencebased interventions aimed at encouraging the consumption of minimally processed foods.
\end{abstract}

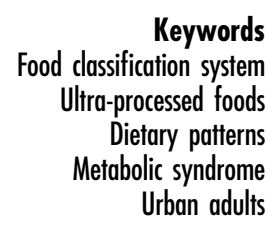

Food processing is defined as the alteration of food from its whole, natural state to enhance its safety, storage, convenience and palatability ${ }^{(1)}$. A number of typologies have been proposed to categorize food products according to their degree of processing ${ }^{(1,2)}$. One commonly used typology is the NOVA food classification system, which was proposed by the School of Public Health of the University of São Paulo, Brazil ${ }^{(3)}$. It assigns foodstuffs to four groups according to the extent and purpose of their industrial processing, including: unprocessed and minimally processed foods (Group 1); processed culinary ingredients (Group 2); processed foods (Group 3); and ultra-processed food and drink (UPF) products (Group $4)^{(3)}$. Compared with unprocessed or minimally processed foods, UPF are typically energy-dense, have a higher glycaemic load and are higher in fat, saturated fat and salt ${ }^{(4,5)}$. Although few studies have explored the direct association between UPF consumption and health markers, available evidence suggests that higher intakes of UPF are associated with the risk for overweight, 
obesity ${ }^{(6,7)}$ and metabolic syndrome (MetS) ${ }^{(8)}$, thus highlighting their potential role in modulating the risk of several diet-related non-communicable diseases.

Reports from countries in Europe and Northern and Central America suggest increasing consumption of $\mathrm{UPF}^{(9-13)}$, which are rapidly displacing staple foods and penetrating all segments of the market ${ }^{(9,11,13-15)}$. Less is known about the intakes of UPF in low-income countries and those undergoing economic and nutrition transition. Within the context of the increasing globalization of food systems, the theory of the nutrition transition suggests that with economic development, the population's consumption pattern shifts from minimally processed diets rich in plant-based staple foods to diets high in meat, fats and processed foods ${ }^{(16)}$. Recent predictions, therefore, propose that the consumption of UPF will continue to increase in developing countries, raising questions about the implications of this trend on the burden of disease in these countries ${ }^{(17-19)}$. This may be of direct relevance to the Eastern Mediterranean region, a region that is witnessing rapid rates of development, mechanization and urbanization, accompanied by dietary shifts and alarming increases in the prevalence of non-communicable diseases $^{(20,21)}$. Lebanon, a small country in the Eastern Mediterranean basin, is no exception to this trend, with non-communicable diseases accounting for over $80 \%$ of the annual deaths nationwide ${ }^{(22)}$.

Given the need to characterize the consumption of UPF in countries of the Eastern Mediterranean region and the scarcity of evidence on the link between UPF consumption and health markers, the present study was undertaken to: (i) estimate the consumption levels of minimally processed, processed and ultra-processed foods in a sample of Lebanese adults; (ii) explore the patterns of intakes from minimally processed, processed and ultra-processed foods in the study population; and (iii) investigate the association of the derived patterns with MetS and its components. In addition, the sociodemographic and lifestyle correlates of the identified patterns were examined. In contrast to previous studies that have used household food expenditure or sales data to assess the consumption of processed foods $^{(5,9,11,13,14,23)}$, the current study was based on an individual dietary survey using a culture-specific FFQ. By characterizing the intakes of processed foods in Lebanon and investigating their association with cardiometabolic risk at the population level, the study findings may inform the development of culture-specific interventions that consider food processing and which may contribute to the prevention of non-communicable diseases in Lebanon and other countries in the Eastern Mediterranean region.

\section{Methods}

Data for the present study were drawn from a communitybased survey conducted among a representative sample of
Lebanese adult participants residing in the Greater Beirut area, which includes the city of Beirut and its suburbs. The survey was carried out between March and May 2014. The survey protocol was approved by the Institutional Review Board of the American University of Beirut and all participants provided written informed consent.

\section{Study participants}

A representative sample of Lebanese adults was selected using a door-to-door sampling strategy and a multistage stratified probability sampling frame. Within this frame, the strata were districts of the Greater Beirut area; within each district, neighbourhoods, then households were selected based on a systematic random sampling ${ }^{(24)}$. The numbers of neighbourhoods and households selected within each district were proportional to the total estimated numbers of neighbourhoods and households within the district, respectively. At the household level, when more than one adult respondent was present, information on the month of birth was obtained and the adult respondent with the most recent month of birth was invited to participate, if eligible. The eligibility criteria were age above 18 years, of Lebanese nationality and residing in the Greater Beirut area. Participants were excluded if they were on dialysis, mentally disabled or pregnant. In addition, given that the original survey was designed to examine the population's exposure to bisphenol A, participants working in plastic or other chemical companies were excluded since they may have been occupationally exposed to bisphenol A. Eligible respondents who agreed to participate in the survey were invited to visit the Department of Nutrition and Food Sciences of the American University of Beirut for data collection. For the present study, the selection of participants from the original survey respondents ( $n$ 501) was undertaken according to the following criteria: (i) having complete anthropometric, biochemical and dietary data; and (ii) with no known diagnosis of chronic disease or any metabolic abnormality. Accordingly, 302 participants were included in the current analysis. The availability of 302 participants allowed the determination of an OR of 2.15 of MetS in relation to the adherence to a certain dietary pattern, at a power of $80 \%$ and $5 \%$ significance. These calculations were made under the assumption of a $15 \%$ difference in exposure between participants with and without MetS ${ }^{(25)}$.

\section{Data collection}

The participants were instructed to visit the Department of Nutrition and Food Sciences after fasting for $10 \mathrm{~h}$ and to bring with them all the medications that they were taking at the time of the study. During their visit, the study participants completed a multicomponent questionnaire and an FFQ during a one-on-one interview. In addition, anthropometric measurements, including weight, height and waist circumference, were obtained, and blood samples were drawn by a licensed phlebotomist. 
Data collection was conducted by trained staff to minimize interviewer and measurement errors. Other quality control measures were applied, including the pre-testing of the study instruments, equipment and data collection procedure, as well as the field monitoring of data collection. The interview and data collection for each participant lasted approximately $1 \mathrm{~h}$.

\section{Sociodemographic, anthropometric and biochemical assessment}

Using the multicomponent questionnaire, information was obtained about the participants' age (in years), sex, marital status (married, including those living with a partner, $v$. single, including widowers and divorcees), area of residence (outside Beirut city $v$. Beirut), level of education (elementary to intermediate, secondary or technical, and university or above), monthly income (in Lebanese Liras (LL)), smoking status (non-smoker, including participants who never smoked, $v$. past and current smokers) and physical activity, which was assessed using the short version of the International Physical Activity Questionnaire (IPAQ). Three categories of physical activity (low, moderate and high) were assigned based on MET-min/week, as follows: low, <600; moderate, at least 600; and high, at least $3000^{(26)}$ (where $\mathrm{MET}=$ metabolic equivalent of task). Data about past medical history and current health status were also obtained.

Anthropometric measurements were taken using standardized protocols ${ }^{(27)}$ and calibrated equipment. Height and body weight were measured using a portable stadiometer (Holtain, Crymych, UK) and a calibrated electronic weighing scale (Seca, Hamburg, Germany), respectively. Participants were weighed to the nearest $0 \cdot 1 \mathrm{~kg}$ in light indoor clothing and with bare feet or stockings. Height was measured without shoes and recorded to the nearest $0.5 \mathrm{~cm}$. All anthropometric measurements were taken twice and the average of the two values was used. BMI was calculated as the ratio of weight (kilograms) to the square of height (metres). Sitting blood pressures, both systolic and diastolic, were obtained twice using a digital sphygmomanometer, at $10 \mathrm{~min}$ intervals.

Using the collected blood samples, fasting glucose levels were determined using an enzymatic method (Cobas 6000; Roche, Indianapolis, IN, USA) and levels of TAG and HDL cholesterol (HDL-C) were measured using an enzymatic spectrophotometric technique using a Vitros 350 analyser (Ortho-Clinical Diagnostics, Johnson \& Johnson, High Wycombe, UK). MetS was diagnosed based on the harmonized definition from the International Diabetes Federation $^{(28)}$, whereby participants were classified as having the MetS if they had three of the five following cardiometabolic risk factors: (i) elevated TAG level $(\geq 150 \mathrm{mg} / \mathrm{dl})$; (ii) low HDL-C level $(<40 \mathrm{mg} / \mathrm{dl}$ for men, $<50 \mathrm{mg} / \mathrm{dl}$ for women); (iii) elevated blood pressure (systolic $\geq 130 \mathrm{mmHg}$ and/or diastolic $\geq 85 \mathrm{~mm} \mathrm{Hg}$ ); (iv) elevated fasting glucose level ( $\geq 100 \mathrm{mg} / \mathrm{dl}$ ); and (v) elevated waist circumference ( $\geq 94 \mathrm{~cm}$ for men, $\geq 80 \mathrm{~cm}$ for women).

\section{Dietary intake assessment and derivation of dietary patterns}

Dietary intake assessment was performed using a culturespecific eighty-item semi-quantitative FFQ which referred to the participants' dietary intake over the previous year ${ }^{(29)}$. To be included in the present study, participants had to have $100 \%$ completion of all questions included in the FFQ. This criterion was met by all participants, given that data collection was interviewer-based and FFQ were checked for completeness after the interview. Participants were asked to record the frequency of their food and beverage consumption per day, per week, per month, per year or never. Participants had the choice to report their intakes in terms of a reference portion size or in grams. The reference portions of the two-dimensional food portion visual ${ }^{(30)}$ were used to assist in portion size estimation, in addition to common household measures. The reported frequency of consumption of each food item and beverage was then converted to daily intake. Total energy intake (TEI) was computed using the food composition database of the Nutritionist Pro ${ }^{\mathrm{TM}}$ software (Axxya Systems LLC, Stafford, TX, USA). The food items listed in the FFQ were grouped into twenty-five food groups based on the NOVA food classification system. The total consumption for each food group was determined by summing the daily portion intake of each item within the group. Using these twenty-five food groups, exploratory factor analysis was implemented to identify patterns of dietary intake. The rotated factor loadings matrix was extracted (Varimax rotation). The derived dietary patterns were labelled based on food groups having a rotated factor loading greater than 10.4l. The factor scores were calculated using multiple regression and were categorized, based on the total sample distribution, into first, second, third and fourth quartiles corresponding to low (first quartile) and medium/high adherence levels (second, third and fourth quartiles).

\section{Statistical analysis}

Frequencies, means and standard deviations were used to describe the various sociodemographic, lifestyle, anthropometric and clinical characteristics of the overall study population and by MetS status. Categorical and continuous variables were compared between participants with and without MetS using $\chi^{2}$ and $t$ tests, respectively. For the twenty-five food groups considered, percentage contribution to TEI was calculated as mean and standard deviation. Multiple logistic regression analyses were used to examine the associations of dietary patterns with MetS and each of its components. For each pattern, six independent regression models were built whereby the dependent variables were MetS and its five components and the independent variable was the adherence to the pattern (low $v$. medium/high). The latter was grouped as such to examine the odds of MetS among participants with low adherence to a certain pattern (belonging to the first quartile) compared with those with higher adherence 
(belonging to the second, third and fourth quartiles) to this pattern. Analyses for model 1 were adjusted for all sociodemographic and lifestyle characteristics considered in the study (age, sex, marital status, area of residence, level of education, income, smoking status and physical activity) as well as TEI. Model 2 included BMI in addition to all the variables included in model 1 .

Multiple logistic regression analyses were also used to assess the sociodemographic and lifestyle correlates of the dietary patterns, using the sociodemographic and lifestyle characteristics as independent variables and adherence to each of the identified patterns as the dependent variables (low (first quartile) $v$. medium/high (second, third and fourth quartiles)). $P<0.05$ indicated statistical significance. The statistical software package IBM SPSS Statistics for Windows version 22.0 was used for data cleaning, management and analysis.

\section{Results}

The sociodemographic, lifestyle and anthropometric characteristics, and cardiometabolic risk factors of the study population are presented in Table 1 . Overall, the mean age of the participants was 39.35 (SD 13.84) years, with a higher proportion of female than male participants (61.3 v. 38.7\%). Three out of four participants (76.6\%) were living in the city of Beirut and $15.0 \%$ had a university level of education. Only $12.3 \%$ of the study participants had a monthly income level higher than 3 million LL (approximately \$US 2000). Among lifestyle factors, including smoking and physical activity, $71.5 \%$ were current smokers and $44.4 \%$ had a low level of physical activity. The comparison of participants with and without MetS showed that those with MetS were significantly older (43.41 (sD 14.69) v. 37.25 (sD 12.89) years) and a higher proportion was male $(51.4 v .31 \cdot 8 \%)$. Also, a higher proportion of participants with lower education levels (elementary to intermediate education) was noted among participants with MetS compared with those without MetS (66.4 v. 52.8\%). Higher prevalence rates of elevated fasting blood glucose and TAG levels, low HDL-C level, elevated blood pressure, elevated waist circumference and obesity were noted among participants with MetS than among those without (Table 1).

Table 2 presents the dietary intakes of various food groups in the study population, based on the NOVA food classifications of unprocessed and minimally processed foods, processed culinary ingredients, processed foods and UPF. Listed within the unprocessed and minimally processed foods category were the following food groups: 'water', 'whole and low-fat milk and dairy products', 'fruits, fruit juices (fresh) and vegetable juices', 'vegetables and legumes', 'meat (fish, red meat and poultry)', 'eggs', 'refined/whole grains and pasta', 'Turkish coffee' and 'olives'. The percentage contribution to TEI from this category was $27 \cdot 10 \%$, with 'fruits, fruit juices (fresh) and vegetable juices' having the highest percentage (5.98\%). The processed culinary ingredients category included two food groups: 'olive oil, vegetable oil, butter, ghee and tahini' and 'sugar, honey' and molasses'; this category had a $12.25 \%$ contribution to TEI. The processed foods category contributed $23.83 \%$ to TEI and included 'wine and beer', 'nuts and seeds (roasted and salted)', 'processed cheeses', 'canned foods (vegetables, legumes and fish)' and 'breads'. Within this category, 'breads' had the highest contribution to TEI (18.21\%). The UPF category included 'canned red and luncheon meats', 'pre-fried French fries', 'liquor', 'condiments (mayonnaise, ketchup and mustard)', 'fast-food sandwiches and pizzas', 'chips and salty snacks (including tortillas and pretzels)', 'sweets and sweetened beverages' and 'sausages (including canned)'. The contribution of this category to TEI was the highest, amounting to $36.53 \%$, with the highest percentage coming from 'sweets and sweetened beverages' (15.67\%; Table 2). Comparing participants with MetS and those without, significantly higher contributions to TEI were noted for the 'breads' food group (20.40 v. 17.21\%, respectively) and processed foods category ( $25.83 v .22 .96 \%$, respectively). Analysis of the daily portion intake of the various food groups for all participants and by MetS was also conducted; the results are shown in the Appendix.

Factor analysis revealed two main dietary patterns: 'ultra-processed' and 'minimally processed/processed', which explained 11.79 and $10.65 \%$ of the dietary intake variance, respectively (Table 3 ). Factor loadings showed that the 'ultra-processed' pattern was characterized by high intakes of: pre-fried French fries; condiments; fastfood sandwiches and pizzas; chips and salty snacks; sausages; nuts and seeds; sweets and sweetened beverages; canned red and luncheon meats; liquor; low-fat milk and dairy products; and meat (fish, red meat and poultry). The 'minimally processed/processed' pattern consisted mostly of: fruits, fruit juices (fresh) and vegetable juices; vegetables and legumes; canned food (fruits, vegetables, legumes and fish); refined and whole grains; processed cheeses; whole milk and dairy products; sugar, honey and molasses; breads; and olive oil, vegetable oil, butter, ghee and tahini. The factor loading matrix of these patterns is shown in Table 3.

The associations of the derived intake patterns with MetS and its components were examined using multiple logistic regression, with MetS and its five components as dependent variables and adherence to the pattern (low $v$. medium/high) as the independent variable (Table 4). For the 'ultra-processed' pattern, no association was observed with MetS or any of its components. For the 'minimally processed/processed' pattern, a medium/high adherence was associated with $75 \%$ lower odds of having hyperglycaemia $(\mathrm{OR}=0.25$; $95 \% \mathrm{CI} 0.07,0.98)$ and approximately $80 \%$ lower odds of having low HDL-C level $(\mathrm{OR}=0.17 ; 95 \% \mathrm{CI} 0.05,0.60)$ and $\mathrm{MetS}(\mathrm{OR}=0 \cdot 18$; 


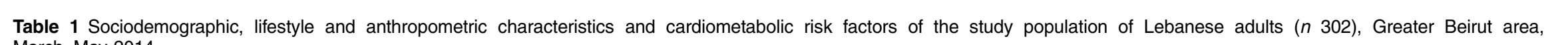
March-May 2014

\begin{tabular}{|c|c|c|c|c|c|c|c|c|}
\hline \multirow[b]{2}{*}{ Variables } & \multicolumn{2}{|c|}{$\begin{array}{l}\text { All participants } \\
(n 302)\end{array}$} & \multicolumn{2}{|c|}{$\begin{array}{l}\text { With metabolic syndrome* } \\
(n \text { 195) }\end{array}$} & \multicolumn{2}{|c|}{$\begin{array}{l}\text { Without metabolic syndrome } \\
(n \text { 107) }\end{array}$} & \multirow[b]{2}{*}{ Significance $\dagger\left(x^{2}\right)$} & \multirow[b]{2}{*}{$P$ value } \\
\hline & Mean or $n$ & SD or $\%$ & Mean or $n$ & SD or $\%$ & Mean or $n$ & SD or $\%$ & & \\
\hline Age (years) & 39.35 & 13.84 & 43.41 & 14.69 & 37.25 & 12.89 & & $1.94 \times 10^{-4}$ \\
\hline \multicolumn{9}{|l|}{ Sex } \\
\hline Male & 117 & 38.7 & 55 & $51 \cdot 4$ & 62 & $31 \cdot 8$ & \multirow[t]{2}{*}{$11 \cdot 190$} & \multirow[t]{2}{*}{0.001} \\
\hline Female & 185 & 61.3 & 52 & 48.6 & 133 & $68 \cdot 2$ & & \\
\hline \multicolumn{9}{|l|}{ Marital status } \\
\hline Single & 109 & $36 \cdot 1$ & 38 & 35.5 & 71 & $36 \cdot 4$ & \multirow{2}{*}{0.024} & \multirow[t]{2}{*}{0.877} \\
\hline Married & 193 & 63.9 & 69 & 64.5 & 124 & 63.6 & & \\
\hline \multicolumn{9}{|l|}{ Area of residence } \\
\hline Outside the city of Beirut & 68 & 23.4 & 21 & $20 \cdot 0$ & 47 & $25 \cdot 4$ & \multirow[t]{2}{*}{1.090} & \multirow[t]{2}{*}{0.296} \\
\hline Beirut city & 222 & 76.6 & 84 & 80.0 & 138 & 74.6 & & \\
\hline \multicolumn{9}{|l|}{ Level of education } \\
\hline Elementary to intermediate & 173 & $57 \cdot 7$ & 71 & $66 \cdot 4$ & 102 & $52 \cdot 8$ & \multirow[t]{3}{*}{8.536} & \multirow[t]{3}{*}{0.014} \\
\hline Secondary or technical & 82 & $27 \cdot 3$ & 28 & $26 \cdot 2$ & 54 & 28.0 & & \\
\hline University & 45 & $15 \cdot 0$ & 8 & 7.5 & 37 & $19 \cdot 2$ & & \\
\hline \multicolumn{9}{|l|}{ Income (LL/month) } \\
\hline$<1.5$ million & 184 & $66 \cdot 4$ & 74 & 71.8 & 110 & 63.2 & \multirow[t]{3}{*}{$2 \cdot 231$} & \multirow[t]{3}{*}{0.328} \\
\hline $1.5-3$ million & 59 & 21.3 & 19 & $18 \cdot 4$ & 40 & 23.0 & & \\
\hline$>3$ million & 34 & $12 \cdot 3$ & 10 & 9.7 & 24 & 13.8 & & \\
\hline \multicolumn{9}{|l|}{ Smoking status } \\
\hline Non-smoker & 86 & 28.5 & 31 & $29 \cdot 0$ & 55 & 28.2 & 0.020 & 0.888 \\
\hline Current smoker & 216 & 71.5 & 76 & 71.0 & 140 & 64.8 & & \\
\hline Physical activity level & & & & & & & & \\
\hline Low intensity & 134 & 44.4 & 52 & 48.6 & 82 & $42 \cdot 1$ & 2.555 & 0.279 \\
\hline Moderate intensity & 96 & 31.8 & 35 & $32 \cdot 7$ & 61 & $31 \cdot 3$ & & \\
\hline High intensity & 72 & $23 \cdot 8$ & 20 & $18 \cdot 7$ & 52 & $26 \cdot 7$ & & \\
\hline Fasting blood glucose (mg/dl) & & & & & & & & \\
\hline Normal & 194 & $64 \cdot 9$ & 32 & $30 \cdot 8$ & 162 & $83 \cdot 1$ & 81.448 & $1.799 \times 10^{-19}$ \\
\hline Elevated $(\geq 100 \mathrm{mg} / \mathrm{dl})$ & 105 & $35 \cdot 1$ & 72 & $69 \cdot 2$ & 33 & $16 \cdot 9$ & & \\
\hline TAG level $(\mathrm{mg} / \mathrm{dl})$ & & & & & & & & \\
\hline Normal & 220 & 73.6 & 48 & $46 \cdot 2$ & 172 & $88 \cdot 2$ & 61.695 & $4.011 \times 10^{-15}$ \\
\hline Elevated $(\geq 150 \mathrm{mg} / \mathrm{dl})$ & 79 & $26 \cdot 4$ & 56 & $53 \cdot 8$ & 23 & $11 \cdot 8$ & & \\
\hline HDL-C level (mg/dl) & & & & & & & & \\
\hline Normal & 183 & $61 \cdot 6$ & 37 & $34 \cdot 6$ & 146 & $76 \cdot 8$ & 51.695 & $6.482 \times 10^{-13}$ \\
\hline Low $($ males $<40 \mathrm{mg} / \mathrm{dl}$, females $<50 \mathrm{mg} / \mathrm{dl}$ ) & 114 & 38.4 & 70 & $65 \cdot 4$ & 44 & $23 \cdot 2$ & & \\
\hline Blood pressure & & & & & & & & \\
\hline No & 217 & 71.9 & 45 & $42 \cdot 1$ & 172 & 88.2 & $72 \cdot 757$ & $1.467 \times 10^{-17}$ \\
\hline Elevated (systolic $\geq 130 \mathrm{mmHg}$ and/or diastolic $\geq 85 \mathrm{mmHg}$ ) & 85 & $28 \cdot 1$ & 62 & 57.9 & 23 & 11.8 & & \\
\hline Waist circumference $(\mathrm{cm})$ & & & & & & & & \\
\hline Normal & 99 & $32 \cdot 8$ & 9 & 8.4 & 90 & $46 \cdot 2$ & $44 \cdot 664$ & $2.339 \times 10^{-11}$ \\
\hline Elevated (males $\geq 94 \mathrm{~cm}$, females $\geq 80 \mathrm{~cm}$ ) & 203 & $67 \cdot 2$ & 98 & 91.6 & 105 & 53.8 & & \\
\hline BMI $\left(\mathrm{kg} / \mathrm{m}^{2}\right)$ & & & & & & & & \\
\hline Not obese $\left(B M I<30 \mathrm{~kg} / \mathrm{m}^{2}\right)$ & 210 & 69.5 & 51 & $47 \cdot 7$ & 159 & 81.5 & 37.426 & $9.494 \times 10^{-10}$ \\
\hline Obese $\left(\mathrm{BMI} \geq 30 \mathrm{~kg} / \mathrm{m}^{2}\right)$ & 92 & 30.5 & 56 & $52 \cdot 3$ & 36 & 18.5 & & \\
\hline
\end{tabular}

LL, Lebanese Lira; HDL-C, HDL cholesterol.

Data are presented as mean and SD for continuous variable (age) or as $n$ and \% for categorical variables.

tSignificance was obtained using the $t$ test and the $x^{2}$ test for continuous and categorical variables, respectively. 
Table 2 Percentage contribution to total energy intake of various food groups in the study population of Lebanese adults ( $n$ 302), Greater Beirut area, March-May 2014

\begin{tabular}{|c|c|c|c|c|c|c|c|c|}
\hline \multirow[b]{2}{*}{ Type of processing } & \multirow[b]{2}{*}{ Type of food } & \multicolumn{2}{|c|}{$\begin{array}{c}\text { All } \\
\text { participants } \\
(n 302) \\
\end{array}$} & \multicolumn{2}{|c|}{$\begin{array}{l}\text { With metabolic } \\
\text { syndrome } \\
(n 107) \\
\end{array}$} & \multicolumn{2}{|c|}{$\begin{array}{l}\text { Without metabolic } \\
\text { syndrome }(n \text { 195) } \\
\end{array}$} & \multirow[b]{2}{*}{$P$ value* } \\
\hline & & Mean & SD & Mean & SD & Mean & SD & \\
\hline \multirow[t]{11}{*}{ Minimally processed foods } & Water & 0 & 0 & 0 & 0 & 0 & 0 & \\
\hline & Whole milk and dairy products & 3.05 & 3.09 & 2.63 & $3 \cdot 13$ & 3.27 & 3.07 & 0.088 \\
\hline & Low-fat milk and dairy products & $2 \cdot 16$ & 3.69 & $2 \cdot 26$ & 4.97 & $2 \cdot 12$ & 2.79 & 0.757 \\
\hline & $\begin{array}{l}\text { Fruits, fruit juices (fresh) and } \\
\text { vegetable juices }\end{array}$ & 5.98 & $5 \cdot 37$ & 5.49 & $4 \cdot 27$ & $6 \cdot 29$ & $5 \cdot 91$ & 0.218 \\
\hline & Vegetables and legumes & 3.89 & $2 \cdot 86$ & 3.51 & $2 \cdot 32$ & 4.08 & 3.07 & 0.095 \\
\hline & Meat (fish, red meat and poultry) & 5.56 & $6 \cdot 10$ & 4.83 & $4 \cdot 15$ & 5.97 & 6.95 & $0 \cdot 122$ \\
\hline & Eggs & 1.09 & 1.28 & 1.25 & 1.48 & 1.01 & $1 \cdot 15$ & 0.131 \\
\hline & Refined/whole grains and pasta & 4.88 & $4 \cdot 14$ & 5.01 & 4.99 & 4.79 & 3.63 & 0.658 \\
\hline & Turkish coffee & 0.03 & 0.08 & 0.03 & 0.06 & 0.03 & 0.09 & 0.739 \\
\hline & Olives & 0.46 & 0.76 & 0.44 & 0.62 & 0.48 & 0.84 & 0.692 \\
\hline & Total & $27 \cdot 10$ & 11.83 & 25.45 & $11 \cdot 28$ & 28.04 & $12 \cdot 26$ & 0.070 \\
\hline \multirow[t]{3}{*}{ Processed culinary ingredients } & $\begin{array}{l}\text { Olive oil, vegetable oil, butter, ghee } \\
\text { and tahini }\end{array}$ & $10 \cdot 42$ & $8 \cdot 38$ & $10 \cdot 85$ & 8.69 & $10 \cdot 17$ & $8 \cdot 21$ & 0.500 \\
\hline & Sugar, honey and molasses & 1.83 & 3.65 & $2 \cdot 30$ & 5.36 & 1.59 & $2 \cdot 23$ & 0.110 \\
\hline & Total & $12 \cdot 25$ & $9 \cdot 13$ & 13.14 & 9.83 & 11.76 & 8.72 & 0.208 \\
\hline \multirow[t]{6}{*}{ Processed foods } & Wine and beer & 0.35 & 1.40 & 0.31 & 1.04 & 0.37 & 1.58 & 0.714 \\
\hline & Nuts and seeds (roasted and salted) & 3.07 & 4.55 & $2 \cdot 84$ & 4.84 & 3.23 & 4.42 & 0.471 \\
\hline & Processed cheeses & 1.32 & 1.89 & 1.38 & 1.53 & 1.29 & $2 \cdot 06$ & 0.582 \\
\hline & Canned food (vegetables, legumes and fish) & 0.88 & $1 \cdot 14$ & 0.90 & 1.36 & 0.86 & 1.01 & 0.768 \\
\hline & Breads & $18 \cdot 21$ & 11.00 & $20 \cdot 40$ & 11.84 & $17 \cdot 21$ & $10 \cdot 32$ & 0.015 \\
\hline & Total & 23.83 & 11.69 & $25 \cdot 83$ & $12 \cdot 07$ & 22.96 & $11 \cdot 28$ & 0.040 \\
\hline \multirow[t]{9}{*}{ Ultra-processed foods } & Canned red and luncheon meats & 0.36 & 0.67 & 0.28 & 0.46 & 0.40 & 0.76 & 0.133 \\
\hline & Pre-fried French fries & 6.91 & 6.73 & 6.34 & 5.92 & 7.29 & $7 \cdot 16$ & 0.242 \\
\hline & Liquor & 0.76 & $3 \cdot 48$ & 0.57 & 1.95 & 0.88 & $4 \cdot 10$ & 0.469 \\
\hline & $\begin{array}{l}\text { Condiments (mayonnaise, ketchup } \\
\text { and mustard) }\end{array}$ & 0.50 & 0.78 & 0.39 & 0.56 & 0.56 & 0.87 & 0.057 \\
\hline & Fast-food sandwiches and pizzas & $8 \cdot 8$ & $6 \cdot 37$ & 8.57 & $6 \cdot 03$ & 8.79 & $6 \cdot 51$ & $0 \cdot 769$ \\
\hline & $\begin{array}{l}\text { Chips and salty snacks (including tortillas } \\
\text { and pretzels) }\end{array}$ & 3.05 & $5 \cdot 10$ & $2 \cdot 29$ & $3 \cdot 85$ & 3.37 & $5 \cdot 57$ & 0.078 \\
\hline & Sweets and sweetened beverages & $15 \cdot 67$ & $11 \cdot 13$ & $16 \cdot 67$ & $12 \cdot 07$ & $15 \cdot 02$ & 10.51 & $0 \cdot 215$ \\
\hline & Sausages (including canned) & 0.49 & $1 \cdot 33$ & 0.34 & 0.57 & 0.56 & 1.61 & 0.163 \\
\hline & Total & 36.53 & 16.51 & 35.45 & 15.97 & 36.87 & $16 \cdot 77$ & 0.473 \\
\hline
\end{tabular}

${ }^{*}$ Significance was derived from the independent $t$ test.

95\% CI 0.04, 0.77). When BMI was added to the multiple logistic regression models, only the association of the 'minimally processed/processed' pattern with hyperglycaemia was attenuated by $2 \%$ and became nonsignificant (Table 4).

To examine the sociodemographic and lifestyle correlates of the dietary patterns, two multiple logistic regression models were built, with an outcome of medium/high $v$. low adherence to the patterns (Table 5). The results showed that age was significantly positively associated with adherence to the 'minimally processed/ processed' pattern $(\mathrm{OR}=1.04 ; 95 \% \mathrm{CI} 1.01,1.07)$ and significantly negatively associated with adherence to the 'ultra-processed' pattern (OR=0.93; 95\% CI 0.90, 0.96). Education level was also significantly associated with both patterns, with a university education level negatively associated with adherence to the 'ultra-processed' pattern $(\mathrm{OR}=0.26 ; 95 \% \mathrm{CI} 0.07,0.87)$ and positively associated with adherence to the 'minimally processed/processed' pattern $(\mathrm{OR}=4.05 ; 95 \%$ CI 1.20, 13.67). Furthermore, compared with non-smokers, current smokers had $50 \%$ lower odds of adhering to the 'minimally processed/ processed' pattern (OR $=0 \cdot 47 ; 95 \%$ CI 0.15, 0.84; Table 5).

A summary of selected findings described in this section was presented elsewhere ${ }^{(31)}$.

\section{Discussion}

Using a food classification system that takes account of the nature, extent and purpose of food processing ${ }^{(3)}$, the present study characterized the consumption of minimally processed, processed and ultra-processed foods in a sample of Lebanese adults and showed that UPF provided one-third of the daily energy intake of adults in Lebanon. The study also explored the overall patterns of intakes of these food groups within the population. It identified two main dietary patterns, the 'ultra-processed' and 'minimally processed/processed', and showed that higher adherence to the 'minimally processed/processed' pattern was significantly associated with lower odds for MetS, hyperglycaemia and low HDL-C level. 
Table 3 Factor loading matrix ${ }^{*}$ of the dietary patterns, derived using factor analysis, among the study population of Lebanese adults ( $n$ 302), Greater Beirut area, March-May 2014

\begin{tabular}{|c|c|c|}
\hline & $\begin{array}{l}\text { 'Ultra- } \\
\text { processed' }\end{array}$ & $\begin{array}{l}\text { 'Minimally } \\
\text { processed/ } \\
\text { processed' }\end{array}$ \\
\hline Pre-fried French fries & 0.72 & - \\
\hline $\begin{array}{l}\text { Condiments (mayonnaise, } \\
\text { ketchup and mustard) }\end{array}$ & 0.65 & - \\
\hline Fast-food sandwiches and pizzas & 0.52 & - \\
\hline $\begin{array}{l}\text { Chips and salty snacks (including } \\
\text { tortillas and pretzels) }\end{array}$ & 0.48 & -0.21 \\
\hline Sausages (including canned) & 0.44 & - \\
\hline $\begin{array}{l}\text { Nuts and seeds (roasted and } \\
\text { salted) }\end{array}$ & 0.44 & 0.20 \\
\hline $\begin{array}{l}\text { Sweets and sweetened } \\
\text { beverages }\end{array}$ & 0.43 & 0.34 \\
\hline Canned red and luncheon meats & 0.41 & 0.24 \\
\hline Liquor & 0.40 & - \\
\hline Low fat-milk and dairy products & 0.31 & - \\
\hline Meat (fish, red meat and poultry) & 0.30 & 0.25 \\
\hline Eggs & 0.27 & - \\
\hline $\begin{array}{l}\text { Fruits, fruit juices (fresh) and } \\
\text { vegetable juices }\end{array}$ & - & 0.59 \\
\hline Vegetables and legumes & - & 0.58 \\
\hline $\begin{array}{l}\text { Canned food (fruits, vegetables, } \\
\text { legumes and fish) }\end{array}$ & - & 0.55 \\
\hline Refined and whole grains & - & 0.53 \\
\hline Processed cheeses & - & 0.50 \\
\hline Whole milk and dairy products & 0.25 & 0.37 \\
\hline Sugar, honey and molasses & - & 0.35 \\
\hline Breads & 0.26 & 0.33 \\
\hline Wine and beer & 0.32 & 0.32 \\
\hline $\begin{array}{l}\text { Olive oil, vegetable oil, butter, } \\
\text { ghee and tahini }\end{array}$ & - & 0.30 \\
\hline Olives & - & 0.25 \\
\hline Water & - & 0.25 \\
\hline Turkish coffee & - & 0.18 \\
\hline Variance explained (\%) & 11.79 & 10.65 \\
\hline
\end{tabular}

*Loadings $<|0.1|$ were removed for simplicity.

When compared with estimates reported from other countries using the same food classification system, the intake of UPF in Lebanon (36.53\% of TEI) exceeded that reported in another developing country, Brazil $(29.6 \%)^{(11)}$, while being lower than estimates described in developed countries such as the USA (57.9\%), Canada $(55 \%)$ and Norway $(49 \%)^{(9,14,15)}$. These findings are in agreement with previous reports concluding that the consumption of convenient, ultra-processed and ready-toeat foods is currently the greatest in developed, highincome countries, while also predicting the gradual establishment of similar consumption patterns in developing countries given the ongoing nutrition transition, increased food systems globalization and the adoption of aggressive marketing strategies for this type of food ${ }^{(16)}$. Compared with data reported in other countries, the consumption of the processed foods category was the highest in Lebanon $(23.83 \quad v \cdot 2 \cdot 5-9.4 \%$ of TEI $)^{(1,9,11,13-15,23)}$. This may be explained by the fact that in the present study, bread, which is considered a main staple food in Lebanon, was included in the processed food category, and not in the UPF group, given that
Lebanese breads do not contain additives and are purchased fresh from bakeries rather than as pre-packaged food products ${ }^{(32)}$. The study findings also show that the consumption of unprocessed and minimally processed foods and processed culinary ingredients combined provided approximately $40 \%$ of TEI in Lebanon, a value that falls within the range reported in several developed countries $(32 \cdot 5-55 \%)^{(1,9,11,13-15,23)}$. This suggests that, in line with the theory of nutrition transition and globalization of the food system, home-based food preparations and cooking are becoming less dominant in the dietary habits of Lebanese adults.

It is important to note that the comparison of data stemming from the present study with those reported from other countries should be interpreted with caution, since in many instances the studies may have differed in dietary assessment methodologies. While the present study was based on an individual dietary survey, most of the other studies were based on household expenditures, retailbased sales or per capita food availability data ${ }^{(9,11,13,14)}$, which may not reflect actual food consumption at the individual level ${ }^{(33)}$

Beyond the characterization of the consumption of unprocessed, processed and ultra-processed foods in terms of contribution to energy intake, the current study explored overall patterns of intakes of these foods and their associations with cardiometabolic risk. This approach of examining overall dietary patterns is receiving increasing attention in nutrition research as it may better reflect the intakes of foods as normally consumed. This approach may also overcome the limitations of the traditional methods of examining single foods or food groups, thus allowing investigation of the joint effects of multiple dietary components on disease risk ${ }^{(34,35)}$. The study findings revealed two main dietary patterns, the 'ultra-processed' and the 'minimally processed/processed' patterns, which together explained almost $22 \%$ of the dietary intake variance. As its name implies, the 'ultra-processed' pattern was characterized by high intakes of convenient, ready-to-eat food items, while also including meat (fish, red meat and poultry), roasted nuts and seeds, and low-fat dairy products. The 'minimally processed/processed' pattern included processed foods such as breads and canned food, but was mainly characterized by high intakes of minimally processed, plant-based foods such as fruits, vegetables, legumes and grains. As such, the 'minimally processed/processed' pattern shared many of the characteristics of the traditional Lebanese dietary pattern, which was previously described as a variant of the Mediterranean diet ${ }^{(36)}$.

The study findings did not indicate a direct association between the 'ultra-processed' pattern and MetS or any of its components. While other studies have examined the association of cardiometabolic risk with the intake of UPF as an a priori set food group ${ }^{(7,8,17)}$, the method adopted in the present study allowed for the examination of food 


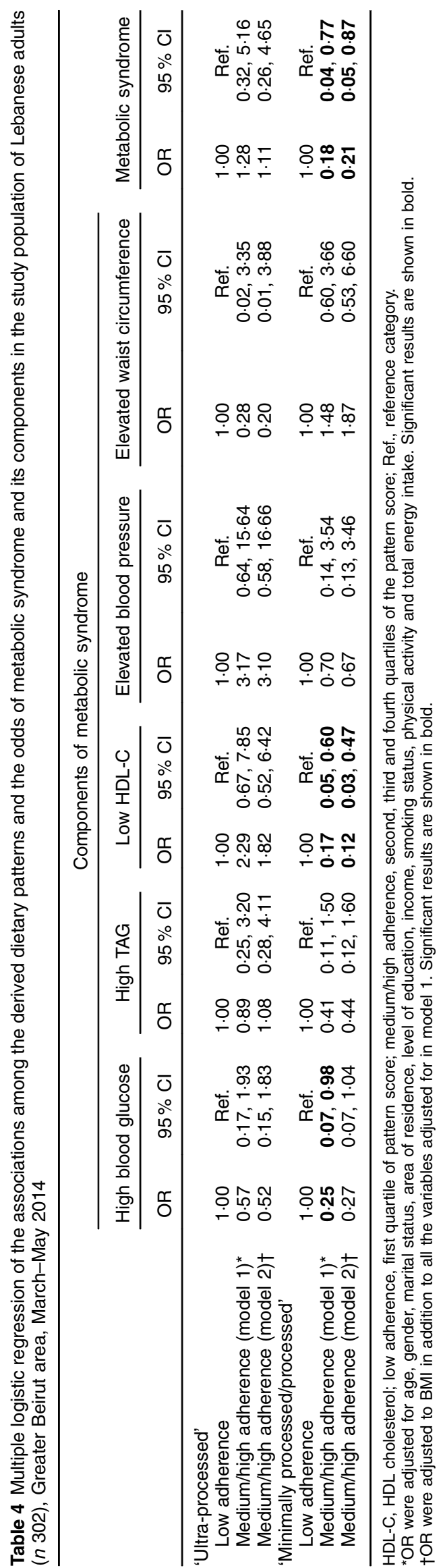

group intake patterns as normally consumed by the population. The fact that the 'ultra-processed' pattern included nuts and seeds, fish and low-fat dairy products in addition to UPF may have diluted the association between UPF and metabolic abnormalities. Interestingly, the present results showed that a higher adherence to the "minimally processed/processed' dietary pattern was associated with lower odds of having hyperglycaemia, low HDL-C level and MetS. In contrast to UPF, minimally processed foods tend to retain their nutritional properties and characteristics. As such, the beneficial combinations of phytochemicals, antioxidants, fibre and monounsaturated fats brought by legumes, fruits, vegetables, grains and olives/olive oil, which are among the main characteristics of the 'minimally processed/processed' pattern, may work in concert to decrease oxidative stress, temper the inflammatory response, enhance fat oxidation, buffer lipid and insulin fluctuations and peaks, improve insulin sensitivity and decrease cardiometabolic risk ${ }^{(37-41)}$. The observed protective association of a diet rich in minimally processed foods and low in UPF against MetS has public health implications given the high prevalence of cardiometabolic risk factors among Lebanese adults. In agreement with the results of the present study, a previous national study showed that $34 \cdot 6 \%$ of healthy Lebanese adults had MetS ${ }^{(42)}$.

The current study also examined the sociodemographic and lifestyle correlates of the identified dietary patterns. The results showed that older age and higher educational levels were associated with increased adherence to the 'minimally processed/processed' dietary pattern. These findings agree with those reported by previous studies conducted in Lebanon and elsewhere, where adherence to 'traditional' dietary patterns, which are typically characterized by higher intakes of minimally processed foods and lower consumption of UPF, tended to increase with age and with level of education ${ }^{(43-47)}$. Our study also showed that, compared with non-smokers, current smokers had significantly lower adherence to the "minimally processed/processed' pattern. These findings may be viewed as a recurrent manifestation of the welldocumented clustering of behavioural risk factors, including smoking and unbalanced $\operatorname{diet}^{(48)}$, a clustering that is increasingly common in countries undergoing the nutrition transition, including Lebanon and other countries in the Eastern Mediterranean region ${ }^{(20)}$.

The results of the present study should be considered in light of the following limitations. First, the findings of the study cannot infer causality given its cross-sectional design. Hence, the significant study findings are reflective of associational relationship between exposure and outcome and cannot be used to show a cause-effect relationship. However, to decrease possible reverse causation, participants who reported the diagnosis of a chronic disease or metabolic abnormalities that may have affected their dietary habits were excluded from the study. 
Table 5 Sociodemographic, lifestyle and anthropometric correlates of the identified dietary patterns, assessed using multiple logistic regression*, in the study population of Lebanese adults $(n 302)$, Greater Beirut area, March-May 2014

\begin{tabular}{|c|c|c|c|c|}
\hline & \multicolumn{4}{|c|}{ Adherence to dietary pattern (medium and high adherence $v$. low) } \\
\hline & \multicolumn{2}{|c|}{ 'Ultra-processed’ } & \multicolumn{2}{|c|}{ 'Minimally processed/processed' } \\
\hline & OR & $95 \% \mathrm{Cl}$ & OR & $95 \% \mathrm{Cl}$ \\
\hline Age & 0.93 & $0.90,0.96$ & 1.04 & $1.01,1.07$ \\
\hline \multicolumn{5}{|l|}{ Sex } \\
\hline Female & 0.78 & $0.31,1.94$ & 0.66 & $0.29,1.51$ \\
\hline \multicolumn{5}{|l|}{ Marital status } \\
\hline Married & 0.92 & $0 \cdot 39,2 \cdot 13$ & 0.76 & $0.36,1.58$ \\
\hline \multicolumn{5}{|l|}{ Area of residence } \\
\hline Beirut & 0.46 & $0.20,1.04$ & $1 \cdot 30$ & $0.58,2.89$ \\
\hline \multicolumn{5}{|l|}{ Level of education } \\
\hline Secondary or technical & 0.78 & $0.31,1.99$ & 1.84 & $0.81,4.17$ \\
\hline University & 0.26 & $0.07,0.87$ & 4.05 & $1.20,13.67$ \\
\hline \multicolumn{5}{|l|}{ Income (LL/month) } \\
\hline 1.5-3 million & 0.63 & $0.25,1.55$ & 0.88 & $0.38,2.04$ \\
\hline$>3$ million & 1.94 & $0.47,8.02$ & 1.12 & $0.34,3.71$ \\
\hline \multicolumn{5}{|l|}{ Smoking } \\
\hline Current smoker & 0.90 & $0.41,1.94$ & 0.47 & $0.15,0.84$ \\
\hline \multicolumn{5}{|l|}{ Physical activity level } \\
\hline Moderate intensity & 0.92 & $0.41,2.09$ & 1.68 & $0.77,3.70$ \\
\hline High intensity & 0.62 & $0.25,1.51$ & 0.64 & $0.30,1.34$ \\
\hline
\end{tabular}

LL, Lebanese Lira.

${ }^{*} \mathrm{OR}$ and $95 \% \mathrm{Cl}$ were derived from multiple regression models. For each pattern, a logistic regression model was built with all the variables listed in the table as independent variables while the dependent variable was low adherence (first quartile) $v$. medium/high adherence (second, third and fourth quartiles) to the pattern. Significant results are shown in bold.

Second, examining the associations between various dietary patterns with MetS and its components in the current study necessitated multiple statistical testing. Therefore, the possibility of a type I error or detection of false positive results cannot be ruled out. Third, the study was based on the use of an FFQ for the assessment of dietary intake, which may be limited by measurement errors, reliance on memory and the number of food items included in the food list ${ }^{(49)}$. However, in the present study, the level of detail of the food list allowed the disaggregation of several food items according to their level of processing. For instance, the questionnaire allowed respondents to distinguish between the consumption of processed meat $v$. unprocessed meat, canned vegetables $v$. fresh vegetables, canned fish $v$. fresh fish, etc. Furthermore, for some foods in the food list, additional probing questions were used to obtain additional information about the foods usually consumed by the individual. Similarly, and to attenuate the restriction imposed by the fixed food list of the FFQ, a question about 'other foods usually consumed' was asked at the end of the interview. It is important to note that although the FFQ used in the present study was not validated in our study population, it was previously used for the assessment of dietary patterns and their relationship with obesity and MetS among Lebanese adults and has yielded plausible findings ${ }^{(42,50)}$. Despite the potential limitations of the FFQ method, it has been shown to be one of the most suitable dietary assessment tools in large epidemiological studies as it provides information on the participants' habitual diet over longer periods of time ${ }^{(51)}$. The FFQ used in the present study was administered by trained nutritionists rather than being self-administered. This approach provides several advantages, as self-administration of the FFQ requires a literate population, and may result in inconsistent interpretations of the food list and lower response and completion rates, each of which may jeopardize the validity of the data ${ }^{(51)}$. However, as observed in most questionnairebased surveys, the interview-based approach may incur a social desirability bias, whereby the survey participants may respond in a manner that they perceive as acceptable or favourable to the interviewer ${ }^{(52)}$. In our study, the fieldworkers who conducted data collection had received extensive training to reduce judgemental verbal and nonverbal communication in order to minimize any social desirability bias. The FFQ and the IPAQ used in the present study were not validated in our population; however, these tools were previously used in dietary and physical assessment studies among Lebanese adults and the findings of these studies yielded plausible associations with obesity, MetS, diabetes and metabolic health ${ }^{(29,42,50,53-59)}$. Finally, the present study was confined to the urban setting of the Greater Beirut area; hence, findings pertinent to the consumption levels of foods may not be representative of less urban settings in the country. The choice of Beirut can be explained by the fact that it comprises $40 \%$ of the Lebanese population and is usually considered a melting pot of the country. 


\section{Conclusion}

In conclusion, to our knowledge, the current study is the first to characterize, based on a food classification system, the consumption of unprocessed, processed and ultra-processed foods in the Eastern Mediterranean region, where data on the intakes of these food categories are completely lacking. The results showed that one-third of the energy intake of adults in Lebanon is derived from UPF, thus highlighting the impact that the globalization of food systems and aggressive UPF marketing strategies may have on dietary habits within the population. The study also identified two main dietary patterns in the population, the 'ultra-processed' and the 'minimally processed/ processed', and documented an inverse association between MetS and adherence to the 'minimally processed/ processed' pattern. These findings may be used for the development of evidence-based interventions and public health strategies aiming to encourage the consumption of minimally processed foods in Lebanon and the region.

\section{Acknowledgements}

Acknowledgements: The authors are grateful to all the study participants for their trust in the research team and to all the volunteer students who provided support during the data collection and ensured the smooth conduct of the study. The research team is grateful to Ms Hikma Shoaib who has developed the Access database for the purposes of the study, thus facilitating data entry and analysis. Financial support: The study was supported by a grant from the National Council for Scientific Research (LNCSR), the University Research Board (American University of Beirut), the Medical Practice Plan (American University of Beirut), the Munib Shahid Fund and the Novo Nordisk development programme. The funders had no role in the design, analysis or writing of this article. Conflict of interest: None of the authors have any personal or financial conflict of interest. Authorship: L.N. and F.N. formulated the research question. L.N. drafted the manuscript and contributed to the interpretation of the data. H.T. led the parent study. M.P.N., N.F.N., H.I. and L.N. provided support in conceiving and designing the parent study. L.I. contributed to data analysis and interpretation. L.N., H.T., M.P.N. and N.F.N. acquired the data. J.A.-R. was involved in coordinating fieldwork, data entry and cleaning. F.N. conducted data analysis and contributed to data interpretation and write up of the manuscript. H.T., M.P.N., N.F.N., H.I. and L.I. critically reviewed the manuscript. All authors guarantee the accuracy of their input and the integrity of this work. Ethics of buman subject participation: Ethical approval was obtained from the Institutional Review Board of the American University of Beirut. Written consent was obtained from all participants.

\section{References}

1. Adams J \& White M (2015) Characterisation of UK diets according to degree of food processing and associations with socio-demographics and obesity: cross-sectional analysis of UK National Diet and Nutrition Survey (2008-12). Int J Behav Nutr Phys Act 12, 160.

2. Moubarac J-C, Parra DC, Cannon G et al. (2014) Food classification systems based on food processing: significance and implications for policies and actions: a systematic literature review and assessment. Curr Obes Rep 3, 256-272.

3. Monteiro C, Cannon G, Levy RB et al. (2012) The Food System. The big issue. World Nutr 3, 527-569.

4. Louzada MLdC, Martins APB, Canella DS et al. (2015) Ultraprocessed foods and the nutritional dietary profile in Brazil. Rev Saude Publica 49, 38.

5. Poti JM, Mendez MA, Ng SW et al. (2015) Is the degree of food processing and convenience linked with the nutritional quality of foods purchased by US households? Am J Clin Nutr 101, 1251-1262.

6. Canella DS, Levy RB, Martins APB et al. (2014) Ultraprocessed food products and obesity in Brazilian households (2008-2009). Plos One 9, e92752.

7. da Costa Louzada ML, Baraldi LG, Steele EM et al. (2015) Consumption of ultra-processed foods and obesity in Brazilian adolescents and adults. Prev Med 81, 9-15.

8. Tavares LF, Fonseca SC, Rosa MLG et al. (2012) Relationship between ultra-processed foods and metabolic syndrome in adolescents from a Brazilian Family Doctor Program. Public Health Nutr 15, 82-87.

9. Moubarac J-C, Batal M, Martins APB et al. (2014) Processed and ultra-processed food products: consumption trends in Canada from 1938 to 2011. Can J Diet Pract Res 75, 15-21.

10. Monteiro CA, Levy RB, Claro RM et al. (2011) Increasing consumption of ultra-processed foods and likely impact on human health: evidence from Brazil. Public Health Nutr 14, $5-13$.

11. Martins APB, Levy RB, Claro RM et al. (2013) Increased contribution of ultra-processed food products in the Brazilian diet (1987-2009). Rev Saude Publica 47, 656-665.

12. Crovetto M \& Uauy R (2012) Changes in processed food expenditure in the population of Metropolitan Santiago in the last twenty years. Rev Med Chil 140, 305-312.

13. Juul F \& Hemmingsson E (2015) Trends in consumption of ultra-processed foods and obesity in Sweden between 1960 and 2010. Public Health Nutr 18, 3096-3107.

14. Solberg SL, Terragni L \& Granheim SI (2016) Ultra-processed food purchases in Norway: a quantitative study on a representative sample of food retailers. Public Health Nutr 19, 1900-2001.

15. Steele EM, Baraldi LG, da Costa Louzada ML et al. (2016) Ultra-processed foods and added sugars in the US diet: evidence from a nationally representative crosssectional study. BMJ Open 6, e009892.

16. Baker P \& Friel S (2014) Processed foods and the nutrition transition: evidence from Asia. Obes Rev 15, 564-577.

17. Monteiro CA, Moubarac JC, Cannon G et al. (2013) Ultra-processed products are becoming dominant in the global food system. Obes Rev 14, 21-28.

18. Stuckler D, McKee M, Ebrahim S et al. (2012) Manufacturing epidemics: the role of global producers in increased consumption of unhealthy commodities including processed foods, alcohol, and tobacco. PLoS Med 9, e1001235.

19. Ng S \& Dunford E (2013) Complexities and opportunities in monitoring and evaluating US and global changes by the food industry. Obes Rev 14, 29-41.

20. Mehio Sibai A, Nasreddine L, Mokdad AH et al. (2010) Nutrition transition and cardiovascular disease risk factors in 
Middle East and North Africa countries: reviewing the evidence. Ann Nutr Metab 57, 193-203.

21. World Health Organization (2008) The Global Burden of Disease 2004. Geneva: WHO.

22. Boutayeb A, Boutayeb S \& Boutayeb W (2013) Multimorbidity of non communicable diseases and equity in WHO Eastern Mediterranean countries. Int J Equity Health 12, 60 .

23. Moubarac J-C, Martins APB, Claro RM et al. (2013) Consumption of ultra-processed foods and likely impact on human health. Evidence from Canada. Public Health Nutr 16, 2240-2248.

24. Central Administration of Statistics (2007) Living Conditions Survey. http://www.cas.gov.lb/index.php/housing-characteri stics-en (accessed November 2013).

25. Dean AG, Sullivan KM \& Soe MM (2013) Open Epi: Open Source Epidemiologic Statistics for Public Health. http://www.openepi.com/SampleSize/SSCC.htm (accessed November 2013).

26. International Physical Activity Questionnaire (2004) Guidelines for Data Processing and Analysis of the International Physical Activity Questionnaire (IPAQ) - Short Form. http:// www.institutferran.org/documentos/scoring_short_ipaq_april04. pdf (accessed November 2013).

27. Lee RD \& Nieman DC (2007) Nutritional Assessment. New York: McGraw-Hill.

28. Alberti K, Eckel RH, Grundy SM et al. (2009) Joint scientific statement. Circulation 120, 1640-1645.

29. Nasreddine L, Hwalla N, Sibai A et al. (2006) Food consumption patterns in an adult urban population in Beirut, Lebanon. Public Health Nutr 9, 194-203.

30. Posner BM, Smigelski C, Duggal A et al. (1992) Validation of two-dimensional models for estimation of portion size in nutrition research. J Am Diet Assoc 92, 738-741.

31. Nasreddine L, Tamim H, Hwalla N et al. (2017) A minimally processed dietary pattern is associated with lower odds of metabolic syndrome among Lebanese adults. FASEB J 31, $788-5$.

32. Toufeili I, Habbal Y, Shadarevian S et al. (1999) Substitution of wheat starch with non-wheat starches and cross-linked waxy barley starch affects sensory properties and staling of Arabic bread. I Sci Food Agric 79, 1855-1860.

33. Food and Agriculture Organization of the United Nations (2015) Guidelines on the collection of information on food processing through food consumption surveys. http://www. fao.org/3/a-i4690e.pdf (accessed August 2016).

34. Cutler GJ, Flood A, Hannan PJ et al. (2012) Association between major patterns of dietary intake and weight status in adolescents. BrJ Nutr 108, 349-356.

35. Newby P \& Tucker KL (2004) Empirically derived eating patterns using factor or cluster analysis: a review. Nutr Rev 62, 177-203.

36. Noah A \& Truswell AS (2001) There are many Mediterranean diets. Asia Pac J Clin Nutr 10, 2-9.

37. Esmaillzadeh A, Kimiagar M, Mehrabi Y et al. (2006) Fruit and vegetable intakes, C-reactive protein, and the metabolic syndrome. Am J Clin Nutr 84, 1489-1497.

38. Sleeth ML, Thompson EL, Ford HE et al. (2010) Free fatty acid receptor 2 and nutrient sensing: a proposed role for fibre, fermentable carbohydrates and short-chain fatty acids in appetite regulation. Nutr Res Rev 23, 135-145.

39. Anderson JW, Baird P, Davis RH et al. (2009) Health benefits of dietary fiber. Nutr Rev 67, 188-205.

40. Fernandez ML \& West KL (2005) Mechanisms by which dietary fatty acids modulate plasma lipids. J Nutr 135, 2075-2078.

41. Lopez S, Bermudez B, Ortega A et al. (2011) Effects of meals rich in either monounsaturated or saturated fat on lipid concentrations and on insulin secretion and action in subjects with high fasting triglyceride concentrations. Am J Clin Nutr 93, 494-499.
42. Naja F, Nasreddine L, Itani L et al. (2013) Association between dietary patterns and the risk of metabolic syndrome among Lebanese adults. Eur J Nutr 52, 97-105.

43. Sánchez-Villegas A, Delgado-Rodriguez M, MartínezGonzález MÁ et al. (2003) Gender, age, socio-demographic and lifestyle factors associated with major dietary patterns in the Spanish Project SUN (Seguimiento Universidad de Navarra). Eur J Clin Nutr 57, 285-292.

44. Naja F, Hwalla N, Itani L et al. (2015) A novel Mediterranean diet index from Lebanon: comparison with Europe. Eur J Nutr 54, 1229-1243.

45. Patino-Alonso MC, Recio-Rodríguez JI, Belio JFM et al. (2014) Factors associated with adherence to the Mediterranean diet in the adult population. J Acad Nutr Diet 114, 583-589.

46. Hu EA, Toledo E, Diez-Espino J et al. (2013) Lifestyles and risk factors associated with adherence to the Mediterranean diet: a baseline assessment of the PREDIMED trial. PLoS One 8, e60166.

47. León-Muñoz LM, Guallar-Castillón P, Graciani A et al. (2012) Adherence to the Mediterranean diet pattern has declined in Spanish adults. J Nutr 142, 1843-1850.

48. Schröder H, Marrugat J, Vila J et al. (2004) Adherence to the traditional Mediterranean diet is inversely associated with body mass index and obesity in a Spanish population. J Nutr 134, 3355-3361.

49. Willett W (1998) Nutritional Epidemiology, 2nd ed. New York: Oxford University Press.

50. Naja F, Nasreddine L, Itani L et al. (2011) Dietary patterns and their association with obesity and sociodemographic factors in a national sample of Lebanese adults. Public Health Nutr 14, 1570-1578.

51. Caan BJ, Lanza E, Schatzkin A et al. (1999) Does nutritionist review of a self-administered food frequency questionnaire improve data quality? Public Health Nutr $\mathbf{2}$, $565-569$.

52. Okamoto K, Ohsuka K, Shiraishi T et al. (2002) Comparability of epidemiological information between self-and interviewer-administered questionnaires. J Clin Epidemiol 55, 505-511.

53. Chamieh MC, Moore HJ, Summerbell C et al. (2015) Diet, physical activity and socio-economic disparities of obesity in Lebanese adults: findings from a national study. BMC Public Health 15, 279.

54. Sibai A-M, Obeid O, Batal M et al. (2008) Prevalence and correlates of metabolic syndrome in an adult Lebanese population. CVD Prev Control 3, 83-90.

55. Sibai AM, Costanian C, Tohme R et al. (2013) Physical activity in adults with and without diabetes: from the "highrisk' approach to the 'population-based' approach of prevention. BMC Public Health 13, 1002.

56. Naja F, Shivappa N, Nasreddine L et al. (2017) Role of inflammation in the association between the western dietary pattern and metabolic syndrome among Lebanese adults. Int J Food Sci Nutr (Epublication ahead of print version).

57. Matta J, Nasreddine L, Jomaa L et al. (2016) Metabolically healthy overweight and obesity is associated with higher adherence to a traditional dietary pattern: a cross-sectional study among adults in Lebanon. Nutrients $\mathbf{8}$, E432.

58. Naja F, Hwalla N, Itani L et al. (2012) Dietary patterns and odds of Type 2 diabetes in Beirut, Lebanon: a case-control study. Nutr Metab (Lond) 9, 111.

59. Jomaa L, Hwalla N, Itani L et al. (2016) A Lebanese dietary pattern promotes better diet quality among older adults: findings from a national cross-sectional study. BMC Geriatr 16, 85 . 
Appendix

Daily portion intake of various food groups in the study population of Lebanese adults (n 302), Greater Beirut area, March-May 2014

\begin{tabular}{|c|c|c|c|c|c|c|c|c|}
\hline \multirow[b]{2}{*}{ Type of processing } & \multirow[b]{2}{*}{ Type of food } & \multicolumn{2}{|c|}{$\begin{array}{c}\text { All } \\
\text { participants } \\
(n \text { 302) }\end{array}$} & \multicolumn{2}{|c|}{$\begin{array}{l}\text { With metabolic } \\
\text { syndrome } \\
\text { (n 107) }\end{array}$} & \multicolumn{2}{|c|}{$\begin{array}{l}\text { Without metabolic } \\
\text { syndrome } \\
(n \text { 195) }\end{array}$} & \multirow[b]{2}{*}{$P$ value } \\
\hline & & Mean & SD & Mean & SD & Mean & SD & \\
\hline \multirow[t]{10}{*}{ Minimally processed foods } & Water & 5.09 & 4.58 & 5.22 & 4.81 & $5 \cdot 00$ & 4.45 & 0.659 \\
\hline & Whole milk and dairy products & 1.01 & 1.23 & 0.90 & 1.22 & 1.07 & $1 \cdot 24$ & 0.250 \\
\hline & Low-fat milk and dairy products & 0.58 & 0.85 & 0.56 & 0.79 & 0.60 & 0.89 & 0.647 \\
\hline & $\begin{array}{l}\text { Fruits, fruit juices (fresh) and } \\
\text { vegetable juices }\end{array}$ & 2.58 & $2 \cdot 16$ & $2 \cdot 59$ & 1.93 & $2 \cdot 60$ & $2 \cdot 29$ & 0.957 \\
\hline & Vegetables and legumes & $3 \cdot 16$ & 2.50 & $3 \cdot 18$ & $2 \cdot 57$ & $3 \cdot 17$ & 2.48 & 0.965 \\
\hline & Meat (fish, red meat and poultry) & 1.60 & 2.55 & 1.42 & 1.71 & $1 \cdot 71$ & 2.92 & 0.350 \\
\hline & Eggs & 0.43 & 0.56 & 0.49 & 0.63 & 0.40 & 0.52 & 0.205 \\
\hline & Refined/whole grains and pasta & 2.00 & 2.02 & 2.04 & $2 \cdot 13$ & 1.97 & 1.81 & 0.779 \\
\hline & Turkish coffee & $1 \cdot 71$ & 3.95 & 1.80 & 3.62 & 1.69 & $4 \cdot 14$ & 0.811 \\
\hline & Olives & 0.38 & 0.64 & 0.39 & 0.65 & 0.38 & 0.63 & 0.814 \\
\hline \multirow[t]{2}{*}{ Processed culinary ingredients } & $\begin{array}{l}\text { Olive oil, vegetable oil, butter, } \\
\text { ghee and tahini }\end{array}$ & $9 \cdot 27$ & 8.5 & $10 \cdot 16$ & $9 \cdot 47$ & 8.84 & $7 \cdot 96$ & 0.197 \\
\hline & Sugar, honey and molasses & 1.31 & 5.91 & 2.07 & $9 \cdot 84$ & 0.90 & $1 \cdot 17$ & 0.103 \\
\hline \multirow[t]{5}{*}{ Processed foods } & Wine and beer & 0.13 & 0.52 & 0.11 & 0.34 & 0.14 & 0.59 & 0.565 \\
\hline & Nuts and seeds (roasted and salted) & 0.43 & 0.71 & 0.43 & 0.77 & 0.44 & 0.68 & 0.900 \\
\hline & Processed cheeses & 0.25 & 0.39 & 0.28 & 0.38 & 0.24 & 0.39 & 0.294 \\
\hline & $\begin{array}{l}\text { Canned food (vegetables, } \\
\text { legumes and fish) }\end{array}$ & 0.37 & 0.57 & 0.36 & 0.54 & 0.37 & 0.59 & 0.950 \\
\hline & Breads & $9 \cdot 28$ & $7 \cdot 15$ & $10 \cdot 30$ & 7.09 & 8.82 & $7 \cdot 14$ & 0.086 \\
\hline \multirow[t]{8}{*}{ Ultra-processed foods } & Canned red and luncheon meats & 0.07 & 0.15 & 0.06 & 0.12 & 0.08 & $0 \cdot 16$ & 0.465 \\
\hline & Pre-fried French fries & $1 \cdot 18$ & 1.44 & 1.05 & 1.05 & $1 \cdot 26$ & 1.63 & 0.237 \\
\hline & Liquor & 0.52 & $2 \cdot 76$ & 0.34 & 1.27 & 0.63 & 3.33 & 0.382 \\
\hline & $\begin{array}{l}\text { Condiments (mayonnaise, ketchup } \\
\text { and mustard) }\end{array}$ & $0 \cdot 84$ & $1 \cdot 71$ & 0.60 & 0.89 & 0.98 & $2 \cdot 02$ & 0.069 \\
\hline & Fast-food sandwiches and pizzas & $2 \cdot 90$ & 2.95 & $2 \cdot 83$ & $2 \cdot 47$ & 2.93 & $3 \cdot 21$ & 0.760 \\
\hline & $\begin{array}{l}\text { Chips and salty snacks (including } \\
\text { tortillas and pretzels) }\end{array}$ & 1.02 & 1.88 & 0.81 & $1 \cdot 37$ & $1 \cdot 13$ & $2 \cdot 10$ & $0 \cdot 160$ \\
\hline & Sweets and sweetened beverages & 6.03 & $6 \cdot 13$ & 6.54 & $7 \cdot 40$ & $5 \cdot 73$ & $5 \cdot 34$ & 0.279 \\
\hline & Sausages (including canned) & $0 \cdot 10$ & 0.46 & 0.05 & 0.09 & 0.13 & 0.57 & 0.167 \\
\hline
\end{tabular}

